\title{
64. SOBRE LAS COMUNIDADES CON POA BULBOSA (POACEAE) DEL MEDIO ATLAS CENTRAL (MARRUECOS)
}

\author{
Antonio GALÁN DE MERA y José A. VICENTE ORELLANA
}

About the communities with Poa bulbosa (Poaceae) in the Middle Central Atlas (Morocco).

Palabras clave. Poetea bulbosae, Marruecos.

Key Words. Poetea bulbosae, Morocco.

Las comunidades con Poa bulbosa L. (Poetea bulbosae Rivas Goday \& RivasMartínez 1978, Poetalia bulbosae Rivas Goday \& Rivas-Martínez 1963) han sido ampliamente tratadas en Europa occidental (Rivas Goday \& Rivas-Martínez, 1963; Rivas Goday, 1964; Rivas Goday \& Ladero, 1970; Rivas Goday \& Rivas-Martínez in Rivas-Martínez, 1978; Rivas-Martínez et al., 1986; Ladero et al., 1992). Sin embargo, en el norte de Africa sólo existen observaciones muy puntuales, en Túnez (Braun-Blanquet, 1953; Debazac, 1958) y en Marruecos (Nègre, 1961; Benabid, 1988). Estos dos últimos trabajos dan a conocer varias asociaciones con Poa bulbosa, siendo Benabid quien hace un intento de jerarquización aunque con deficiencias de orden sintaxonómico que nos llevan a la creación de una nueva alianza atlásica (ver tabla) y a la lectotipificación de cuatro asociaciones.

\section{Potentillo maurae-Campanulion filicaulis all. nova}

(holotypus: Phleo nodosi-Poetum bulbosae Benabid 1988)

Alianza que agrupa a comunidades basófilas (calizas, dolomías y basaltos alcalinos) de óptimo supramediterráneo húmedo (Argyrocytiso battandieri-Cedreto atlanticae sigmetum, Barbero et al., 1981; Finckh \& Deil, 1989) del Medio Atlas Central. Son plantas características: Campanula filicaulis Durieu, Carduncellus pinnatus (Desf.) DC., Carduncellus rhaponticoides Coss. \& Durieu, Carum jahandiezii Litard. \& Maire, Leontodon pitardii Maire, Limonium moureti (Pit.) Maire, Plantago coronopus L. subsp. cupani (Guss.) Nyman, Potentilla maura Th. Wolf, Silene mekinensis Coss., Trifolium humile Ball y Vulpia sicula (C. Presl) Link.

Estas comunidades de Poetea alcanzan grandes extensiones y están favorecidas por el majadeo de grandes rebaños de ovejas organizados en un régimen tradicional de pastoreo nómada.

La alianza Trisetario flavescentisCaricion chaetophyllae dada a conocer por Benabid (1988) se incluye en la sinonimia al no haber elegido asociación tipo y estar descrita sobre un número elevado de características de Molinio-Arrhenatheretea R. Tx. 1937 (artículos 5 y $17, \mathrm{CNF}$ ). La alianza más próxima es $P o o$ bulbosae-Astragalion sesamei Rivas Goday \& Ladero 1970, que reune a las comunidades cespitosas de Poa bulbosa de la Península Ibérica sobre suelos de neutro-básicos a básicos. Por tanto, Potentillo-Campanulion es la única alianza descrita por el momento en Marruecos. Sin embargo, la comunidad

Este trabajo ha sido realizado en el marco del proyecto 12/95 de la Universidad San Pablo-CEU y gracias a la estancia promovida por la Deutscher Akademischer Austauschdienst en la Universidad de Bayreuth (Alemania). 
Potentillo maurae-Campanulion filicaulis all. nova (Poetalia bulbosae, Poetea bulbosae)

$\mathrm{N}$. de inventarios N. de orden

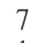

Características de asociaciones

Phleum bertolonii

Leontodon pitardii

Silene mekinensis

Vulpia sicula var. genuina

Carum jahandiezii

Características de Potentillo-Campanulion

Plantago coronopus subsp. cupani

Trifolium humile

Potentilla maura

Campanula filicaulis

Carduncellus rhaponticoides

Carduncellus pinnatus

Limonium moureti

Características de Poetalia y Poetea

Poa bulbosa

Ranunculus millefoliatus

Paronychia argentea

Trifolium subterraneum

Trifolium tomentosum

Ranunculus paludosus

Características de Molinio-Arrhenatheretea

Carex divisa

Festuca rubra

Hieracium pseudopilosella

Trisetum flavescens

Hypochoeris radicata

Taraxacum atlanticum

Lotus corniculatus var. pilosus

Dactylis glomerata

Arrhenatherum elatius subsp. bulbosum

Mentha pulegium

Festuca arundinacea

Cynodon dactylon

Anthoxanthum ovatum

Holcus lanatus

Lolium perenne

Compañeras

Juncus bufonius

Eryngium bourgatii

Convolvulus lineatus

Echinaria capitata

Helianthemum croceum

Eryngium triquetrum

Cerastium gibraltaricum

Evax pygmaea

Herniaria glabra

Caucalis caerulescens

Armeria choulettiana

II

I

i

$\begin{array}{ll}5 & 4\end{array}$

3

4

27

$5-16$

16

123

45

V
I
III
II

$\begin{array}{llllll}\text { III } & \text { III } & 3 & 3 & \text { III } & + \\ \text { I } & \text { II } & 1 & 2 & \text { II } & \text {. } \\ \text { IV } & \text { V } & . & . & \text { V } & \text { I } \\ \text { V } & \text { II } & . & . & \text { IV } & \text { II } \\ \text { III } & . & . & . & \text { IV } & + \\ \text { II } & . & 3 & . & . & . \\ . & \text {. } & 4 & . & \text { r } & \text {. }\end{array}$

V $\quad$ V

II IV

II III

III

III

III

III

. $\mathrm{V}$

III

III

. II

II

\begin{tabular}{|c|c|c|c|c|}
\hline I & II & & 3 & I \\
\hline IV & . & 4 & . & $\mathrm{r}$ \\
\hline IV & . & . & . & I \\
\hline I & . & & . & I \\
\hline I & . & 4 & . & . \\
\hline & III & 2 & . & . \\
\hline $\begin{array}{l}\text { I } \\
\text { V }\end{array}$ & . & & . & \\
\hline $\begin{array}{l}\text { V } \\
V\end{array}$ & i & 4 & . & . \\
\hline $\begin{array}{l}\text { V } \\
\text { I }\end{array}$ & IV & 3 & . & \\
\hline III &. & & $\cdot$ & III \\
\hline
\end{tabular}




\begin{tabular}{|c|c|c|c|c|c|c|}
\hline $\begin{array}{l}\text { N. de inventarios } \\
\text { N. de orden }\end{array}$ & $\begin{array}{l}7 \\
1\end{array}$ & $\begin{array}{l}5 \\
2\end{array}$ & $\begin{array}{l}4 \\
3\end{array}$ & $\begin{array}{l}4 \\
4\end{array}$ & $\begin{array}{l}27 \\
5\end{array}$ & $\begin{array}{l}16 \\
6\end{array}$ \\
\hline Alyssum granatense & III & . & 1 & . & . & . \\
\hline Bupleurum spinosum & II & . & & . & . & + \\
\hline Galium corudifolium & I & . & 1 & . & . & . \\
\hline Cynara histrix & II & . & 3 & . & . & . \\
\hline Bromus hordeaceus & I & . & 1 & . & . & . \\
\hline Anthemis pedunculata & I & . & . & . & & + \\
\hline Catananche caespitosa & . & . & . & . & II & + \\
\hline Erysimum bocconei & . & . & 3 & . & . & III \\
\hline Dasypyrum hordeaceum & . & . & 1 & . & . & II \\
\hline Aegilops geniculata & & & 2 & & I & . \\
\hline Puccinellia distans & & II & . & 2 & & \\
\hline Romulea sp. & & . & . & . & $\mathrm{V}$ & II \\
\hline Thymelaea virgata & . & . & . & . & III & II \\
\hline Convolvulus mazicum & . & . & . & . & II & + \\
\hline Veronica praecox & . & . & . & . & I & I \\
\hline Evacidium discolor & & . & . & . & I & II \\
\hline Veronica rosea & . & . & . & . & + & III \\
\hline
\end{tabular}

Taxones con una sola presencia.- En 1: Scleranthus polycarpos I, Cerastium glomeratum II; en 3: Medicago minima 2, Salvia argentea 2, Trifolium campestre 2; en 4: Hordeum murinum 2; en 5: Colchicum ayanicum I.

Procedencia de los inventarios. 1: Phleo nodosi-Poetum bulbosae (valle de Bekrite, Khénifra- inventarios propios); 2: Phleo nodosi-Poetum bulbosae (depresiones de Afnourir, Aïn Leuh, Azrou- Benabid, 1988); 3: Sileno mekinensis-Caricetum divisae (Tissfoula y Iguer Aouragh, Aïn Leuh, Azrou- Benabid, 1988); 4 : Vulpio genuinae-Limonietum mouretii (Aïn Leuh, Azrou- Benabid, 1988); 5: Caro-Potentilletum maurae (Jbel Saa, Itzer- Nègre, 1961); 6: Poo-Caricetum divisae (Jbel Saa, Itzer- Nègre, 1961).

acidófila de Paronychia echinulata Chater y Plantago serraria L. dada a conocer por Debazac (1958) en Túnez, es incluíble en la alianza mediterránea occidental PeriballioTrifolion subterranei Rivas Goday 1964 (pro nom. inv.).

Las cuatro asociaciones lectotificadas son las siguientes:

\section{1 - Phleo nodosi-Poetum bulbosae (lectotypus:} inv. 5, tabla 14-Benabid, 1988). Comunidad de suelos coluvionarios arcillosos.

2- Sileno mekinensis-Caricetum divisae (lectotypus: inv. 1, tabla 12-Benabid, 1988). Suelos terrosos procedentes de dolomías.

3-Vulpio genuinae-Limonietum mouretii (lectotypus: inv. 2, tabla 13- Benabid, 1988). Comunidad de vertisuelos básicos siendo la más desviante por aumento de la humedad edáfica.

4-Caro-Potentilletum maurae (lectotypus: inv.
3, tabla 3-Nègre, 1961; Sin.: Poo-Caricetum divisae Nègre, 1961). Comunidad de suelos derivados de basaltos alcalinos.

AGRADECIMIENTOS. Gracias al personal del herbario UBT y al Prof. U. Deil, por poner a nuestra disposición su espléndida colección de plantas de Marruecos.

\section{BIBLIOGRAFÍA}

BARBERO, M., P. QUÉZEL \& S. RIVASMARTÍNEZ -1981- Contribution à l'étude des groupements forestiers et préforestiers du Maroc. Phytocoenol. 9(3): 311-412.

BENABID, A. -1988- Contribution à l'étude des groupements non forestiers du Moyen Atlas Central (Maroc). Bull. Inst. Sci. Rabat 12: 7998.

BRAUN-BLANQUET, J. -1953- Irradiations européennes dans la végétation de la Kroumirie. 
Vegetatio 4: 182-194.

DEBAZAC, E.F. -1958- La végétation forestière de la Kroumirie. Ann. Ecol. Nat. Eaux For. Stat. Rech. Exp. 16(1): 1-133.

FINCKH, M. \& U. DEIL -1989- Approches de l'étude de la degradation des fôrets du Jbel Bougriy (Moyen Atlas Central). RGM (n.s.) 13(1): 55-68.

LADERO, M., E. BIONDI, L. MOSSA y A. AMOR -1992-Los pastizales mediterráneos presididos por Trifolium slibterraneum en la isla de Cerdeña (Italia). Doc. Phytosociol. (n.s.) 14: 45-63.

NÈGRE, R. -1961- Les associations végétales du Jbel Saa. Bull. Soc. Sci. Nat. Maroc 41: 19-62.

RIVAS GODAY, S. y S. RIVAS-MARTÍNEZ 1963-Estudio y clasificación de los pastizales españoles. Publ. Ministerio de Agricultura. Madrid.

RIVAS GODAY, S. -1964-Vegetación y flórula de la cuenca extremeña del Guadiana. Publ. Dip. Prov. Badajoz. Madrid.
RIVAS GODAY, S. y M. LADERO ÁLVAREZ 1970- Pastizales cespitosos de Poa bulbosa L. Origen, sucesión y sistemática. Anales R. Acad. Farmacia 36(2): 139-181.

RIVAS-MARTíNEZ, S. -1978-Sur la syntaxonomie des pelouses therophytiques de l'Europe occidentale. Coll. Phytosoc. 6: 55-71.

RIVAS-MARTÍNEZ, S., F. FERNÁNDEZ GONZÁLEZ y D. SÁNCHEZ-MATA -1986Datos sobre la vegetación del Sistema Central y Sierra Nevada. Opusc. Bot. Pharm. Complutensis 2: 1-136.

Aceptado para su publicación en Marzo de 1997

Dirección de los autores. Departamento de Biología. Laboratorio de Botánica. Universidad San PabloCEU. Ap. 67. E-28660 Boadilla del Monte (Madrid). Fax. (91) 3510475 .

\title{
65. ASPECTOS NOMENCLATURALES, ECOLÓGICOS Y BIOGEOGRÁFICOS SOBRE LA RESEDETUM VALENTINAE (ALL. SCROPHULARION SCIOPHILAE)
}

\author{
Manuel B. CRESPO, José Luis SOLANAS, Ana JUAN y Luis SERRA.
}

Nomenclatural, ecological and biogeographical features about Resedetum valentinae (All. Scrophularion sciophilae)

Palabras clave. Sintaxonomía, fitosociología, nomenclatura, Scrophularion sciophilae, Alicante, España.

Key words. Syntaxonomy, phytosociology, nomenclature, Scrophularion sciophilae, Alicante, Spain.

En el contexto de una revisión de las comunidades glareícolas iberolevantinas, $\mathrm{O}$. Bolòs (1974) describió la asociación Resedetum valentinae - Scrophularion sciophilae $\mathrm{O}$. Bolòs 1957, Andryaletalia ragusinae Rivas
Goday \& Rivas Mart. ex O. Bolòs \& Vigo in Folch 1981, Thlaspietea rotundifolii Br.-Bl. 1947 - sobre la base de un inventario tomado en las proximidades de la Font Roja de Alcoi (Alicante), territorio incluido en el subsector 